\title{
Automation of PacBio SMRTbell NGS library preparation for bacterial genome sequencing
}

Nguyet Kong ${ }^{1}$, Whitney $\mathrm{Ng}^{2}$, Kao Thao ${ }^{3}$, Regina Agulto ${ }^{1}$, Allison Weis ${ }^{1}$, Kristi Spittle Kim ${ }^{4}$, Jonas Korlach, Luke Hickey $^{4}$, Lenore Kelly ${ }^{5}$, Stephen Lappin ${ }^{5}$ and Bart C. Weimer ${ }^{1 *}$

\begin{abstract}
Background: The PacBio RS II provides for single molecule, real-time DNA technology to sequence genomes and detect DNA modifications. The starting point for high-quality sequence production is high molecular weight genomic DNA. To automate the library preparation process, there must be high-throughput methods in place to assess the genomic DNA, to ensure the size and amounts of the sheared DNA fragments and final library.

Findings: The library construction automation was accomplished using the Agilent NGS workstation with Bravo accessories for heating, shaking, cooling, and magnetic bead manipulations for template purification.

The quality control methods from gDNA input to final library using the Agilent Bioanalyzer System and Agilent TapeStation System were evaluated.

Conclusions: Automated protocols of PacBio $10 \mathrm{~kb}$ library preparation produced libraries with similar technical performance to those generated manually. The TapeStation System proved to be a reliable method that could be used in a 96-well plate format to QC the DNA equivalent to the standard Bioanalyzer System results. The DNA Integrity Number that is calculated in the TapeStation System software upon analysis of genomic DNA is quite helpful to assure that the starting genomic DNA is not degraded. In this respect, the gDNA assay on the TapeStation System is preferable to the DNA 12000 assay on the Bioanalyzer System, which cannot run genomic DNA, nor can the Bioanalyzer work directly from the 96-well plates.
\end{abstract}

Keywords: PacBio SMRTbell NGS library preparation, Bacterial genomic DNA, Automation, NGS workstation, TapeStation System, Bioanalyzer

\section{Introduction}

Increased throughput from the use of next generation sequencing methods has revealed new information about the function and structure of bacterial genomes. The use of short reads to produce draft genomes leads to problems with GC content bias and repeat regions that make it tedious to produce closed genome assemblies. This technical note discusses the PacBio RS II approach using a single molecule, real-time DNA sequencing approach to improve genome assembly through extra-long read lengths. By reducing the number of contigs, the accuracy

\footnotetext{
* Correspondence: bcweimer@ucdavis.edu

${ }^{1}$ Population Health and Reproduction Department, School of Veterinary

Medicine, University of California-Davis, Davis, CA, USA

Full list of author information is available at the end of the article
}

of the de novo assembly of bacterial whole genomes is facilitated. The real-time technology of the PacBio RS II allows determination of not only the full, closed, gDNA sequence, but also epigenetic modifications and plasmid DNA sequence simultaneously.

The 100K Pathogen Genome Project [1] is using the PacBio $10 \mathrm{~kb}$ SMRTbell Template Preparation kit to produce 1,000 closed genomes. The scale of this project required automation of the construction of the sequencing (SMRTbell ${ }^{\mathrm{Tm}}$ ) library. To prepare libraries for sequencing in this way, gDNA must be cut into fragments to a target size of $10 \mathrm{~kb}$. Critical to generating long sub-reads, it is important to start with high quality gDNA input in order to shear the gDNA into the target fragment size to ensure the correct concentrations 
during library construction to react properly with the concentrations of reagents in each of the given steps. Gel electrophoresis is a low-resolution traditional method with sizing against a ladder and determining concentration on an agarose gel by comparing peak density to a standard, and since it cannot be automated, is not suitable for a project of this size. Another way to measure size and concentration is to use the Agilent 2100 Bioanalyzer with the DNA 12000 assay, but the instrument only runs 12 samples at a time and cannot be automated. We will discuss the automation of preparation of libraries with the SMRTbell Template Preparation kit as well as analysis of gDNA, fragmented DNA and the final libraries ready for sequencing with both the Agilent electrophoresis platform: Agilent 2100 Bioanalyzer System using the DNA 12000 assay and the Agilent TapeStation System using the genomic DNA ScreenTape and matching reagents.

\section{Procedure}

Campylobacter jejuni, Listeria monocytogenes, Vibrio fluvialis and Salmonella enterica serovar. Enteritidis were cultured in appropriate culture medium and growing condition listed in Tables 1 and 2. Bacteria were cultured on the appropriate agar and pellets were made for extraction. DNA was extracted from the cell pellets using a kit and clean-up was accomplished with a spin column [2-4]. Absorbance ratios at 260/280 and 260/230 were measured with a NanoDrop 2000 UV-vis spectrophotometer (Thermo Fisher Scientific, Waltham MA). A Qubit 2.0 Fluorometer (Q32866) was used with a Qubit dsDNA HS Assay Kit (Q32854, both from Invitrogen, Carlsbad CA) to measure the gDNA concentration and confirm DNA input of $10 \mu \mathrm{g}$ before shearing. The initial evaluation of the quantity and size distribution of the purified gDNA was with the Agilent 2200 TapeStation Nucleic Acid System (G2965AA) controlled by Agilent 2200 TapeStation Software A.01.05, using the Agilent Genomic DNA ScreenTape (5067-5365) and the Agilent Genomic DNA Reagents (5067-5366) with samples drawn from a 96-well plate $[5,6]$

Genomic DNA was sheared using the Covaris g-TUBE device (520079) according to the manufacturer specifications [7]. After fragmentation, DNA was evaluated with the TapeStation System with the Genomic DNA assay and also with the Agilent 2100 Bioanalyzer System with the Agilent DNA 12000 assay (5067-1508) [8, 9]. Both of these methods have minimal sample consumption and return both sizing and quantitation. The sheared gDNA sample input was normalized for all samples between 1-5 $\mu \mathrm{g}$ into library construction for PacBio SMRTbell $10 \mathrm{~kb}$ Library Preparation.

The SMRTbell Template Preparation kit from Pacific Biosciences (Menlo Park CA) was used on the Agilent NGS Workstation (G5522A, Agilent Technologies, Santa Clara CA). The workflow to construct the final DNA libraries for sequencing is shown in Fig. 1 and involved automation of these steps:

1. Determination of the quality of the gDNA

2. Fragment gDNA using a Covaris g-TUBE device

3. QC the sizing and adjust the concentration

4. Repair DNA damage and repair ends of fragmented DNA

5. Purify the DNA

6. Blunt-end ligate using blunt adapters

7. Purify template for submission to a sequencer

In Fig. 2, A (Post Shearing Clean-up) and B (10kb Library Prep Runset Dual SPRI) are two of the VWorks protocol graphical user interfaces that help with the NGS Workstation setup and deck layout to optimize the use of reagent volumes. This interface allows the user to view the progress of the procedure. In Fig. 2c, the Excel template assists with laying out the reagent amounts and calculations, and provides a record of each batch of reagents preparation and lot numbers.

With the automation, this workflow takes about $7 \mathrm{~h}$ for post-shearing clean-up and library construction. Once the PacBio $10 \mathrm{~kb}$ library is made, the final library was confirmed with the Agilent 2200 TapeStation with the Genomic DNA ScreenTape assay and the Agilent 2100 Bioanalyzer System with the Agilent DNA 12000 assay to determine the size of the library. Libraries are quantified using a Qubit 2.0 Fluorometer (Q32866) with a Qubit dsDNA HS Assay Kit (Q32854, both from Invitrogen, Carlsbad CA) to measure the library concentration before

Table 1 Organisms used in this study

\begin{tabular}{|c|c|c|c|c|c|c|c|}
\hline ID\# & Color & Bacterium & $\begin{array}{c}\text { GC content } \\
(\%)\end{array}$ & $\begin{array}{c}\text { Gram } \\
\text { Reaction }\end{array}$ & $\begin{array}{l}\text { Approx. } \\
\text { Genome } \\
\text { Size (MB) }\end{array}$ & $\begin{array}{l}\text { Culture } \\
\text { Medium }\end{array}$ & $\begin{array}{c}\text { Growing } \\
\text { Conditions }\end{array}$ \\
\hline 1 & & $\begin{array}{l}\text { Campylobacter } \\
\text { jejuni }\end{array}$ & 30 & Negative & 2 & $\begin{array}{c}\text { Sheep blood } \\
\text { agar }\end{array}$ & $\begin{array}{l}\text { Microaerophilic } \\
37^{\circ} \mathrm{C}\end{array}$ \\
\hline 2 & & $\begin{array}{l}\text { Listeria } \\
\text { monocytogenes }\end{array}$ & 38 & Positive & 2 & $\begin{array}{l}\text { Brain heart } \\
\text { infusion } \\
\text { agar }\end{array}$ & $\begin{array}{l}\text { Aerobic } \\
\quad 37^{\circ} \mathrm{C}\end{array}$ \\
\hline 3 & & Vibrio fluvials & 41 & Negative & 5 & $\begin{array}{l}\text { Trypticase } \\
\text { soy agar }\end{array}$ & $\begin{array}{l}\text { Aerobic } \\
37^{\circ} \mathrm{C}\end{array}$ \\
\hline 4 & & $\begin{array}{l}\text { Salmonella } \\
\text { enterica } \\
\text { serovar } \\
\text { Enteritidis }\end{array}$ & 52 & Negative & 5 & & $\begin{array}{r}\text { Aerobic } \\
37^{\circ} \mathrm{C}\end{array}$ \\
\hline
\end{tabular}


Table 2 gDNA quality, average shearing size and average final library for each bacterium

\begin{tabular}{|c|c|c|c|c|c|c|c|}
\hline \multirow{2}{*}{$\begin{array}{c}\text { ID } \\
\#\end{array}$} & \multirow[t]{2}{*}{ Color } & \multirow[t]{2}{*}{ Bacterium } & \multirow{2}{*}{$\begin{array}{c}\text { DIN } \\
\text { TapeStation }\end{array}$} & \multicolumn{2}{|c|}{ Average Shearing (kb) } & \multicolumn{2}{|c|}{ Average Final Library (kb) } \\
\hline & & & & Bioanalyzer & TapeStation & Bioanalyzer & TapeStation \\
\hline 1 & & C. jejuni & 9.1 & 10 & 11 & 9.1 & 19 \\
\hline 2 & & L. monocytogenes & 9.1 & 13.5 & 12 & 9.5 & 19 \\
\hline 3 & & V. fluvials & 9.3 & 11.6 & 14 & 10 & 25 \\
\hline 4 & & $\begin{array}{c}\text { S. enterica serovar. } \\
\text { Enteritidis }\end{array}$ & 9.5 & 17 & 20 & 15 & 24 \\
\hline
\end{tabular}

submission to the sequencing facility. The sequencing facility anneals sequencing primer and binds polymerase to the SMRTbell templates before loading the library onto the PacBio RS II.

\section{Discussion}

The genomic DNA isolated from four model organisms with a range of $\mathrm{GC}$ content were made into libraries prepared on the Agilent NGS Workstation with PacBio SMRTbell Template Preparation kit for sequencing on the PacBio RSII. Finished sequences showed GC content very close to the known GC content, thus showing this process produced minimal bias (Table 1).
For the best results to produce genomic sequences, it is important the starting material be relatively free of organics and protein, and be at least 50 kilobases to insure long fragments can be obtained for sequencing. The microbes used are listed in Table 1 and include four genera of varying length and GC content. The organisms were cultured and genomic DNA was extracted followed by spin column clean-up. The quality of the gDNA was measured with the NanoDrop and the 260/280 nm and the $260 / 230 \mathrm{~nm}$ ratios were calculated. The $260 / 280 \mathrm{~nm}$ ratio and $260 / 230 \mathrm{~nm}$ ratio of 1.8 was the requirement for further use of each extraction. The Agilent 2200 TapeStation System with the Genomic DNA assay was used to assess size and concentration of each sample as

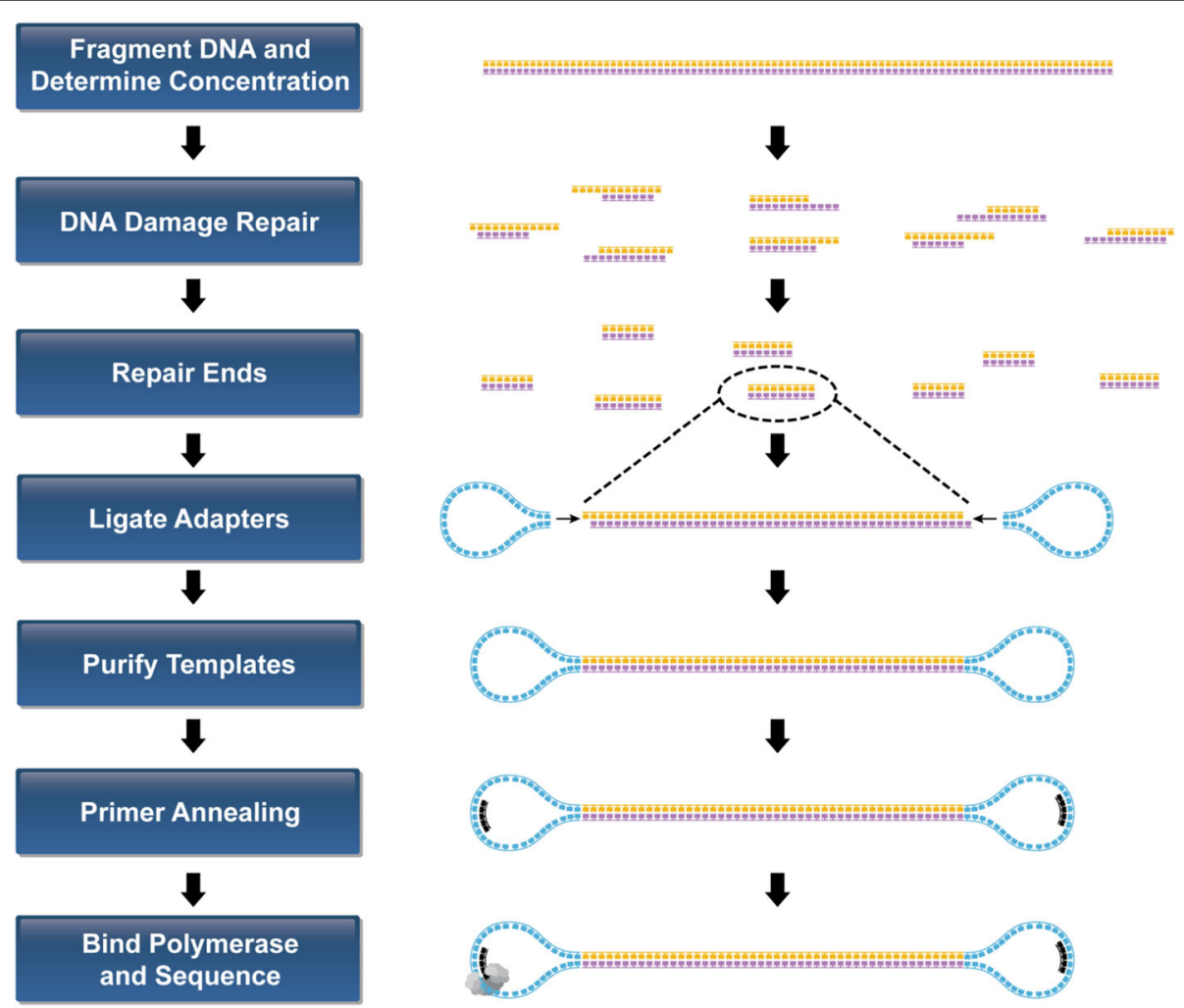

Fig. 1 PacBio SMRTbell Template Preparation Workflow for PacBio RS II system. PacBio SMRTbell Template Preparation Workflow for PacBio RS II system. This workflow is used to prepare libraries from fragmented and concentrated DNA using Covaris g-TUBE and concentrated using the AMPure magnetic beads before following PacBio SMRTbell $10 \mathrm{~kb}$ Library Preparation procedures 


\section{a}

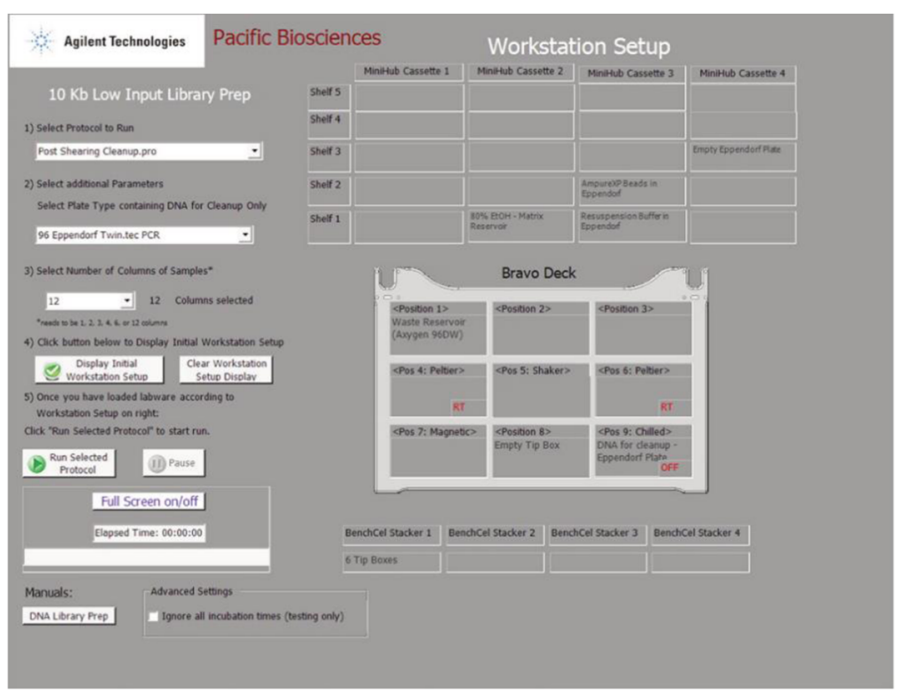

b

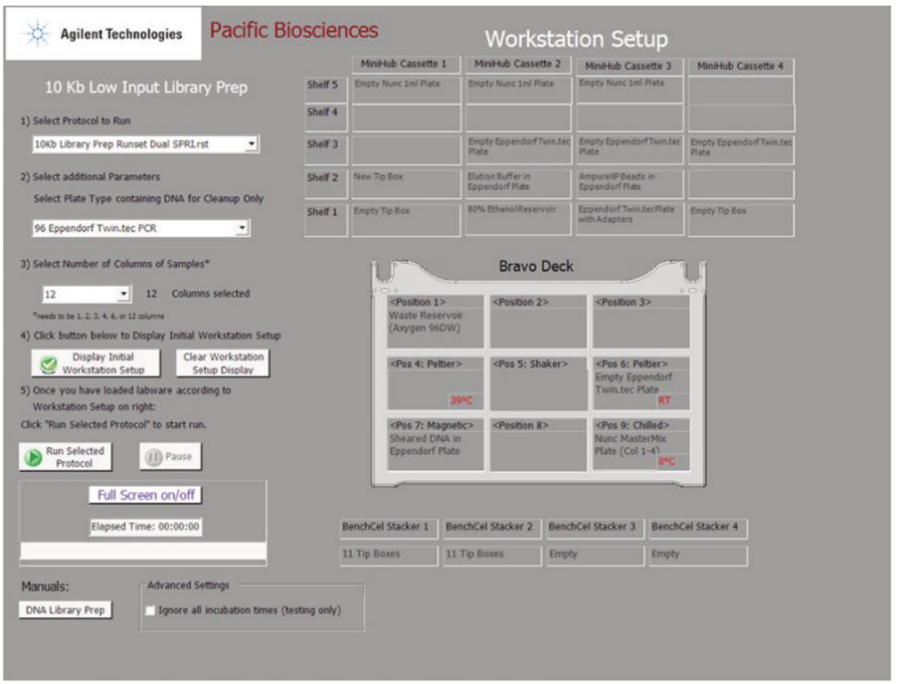

C
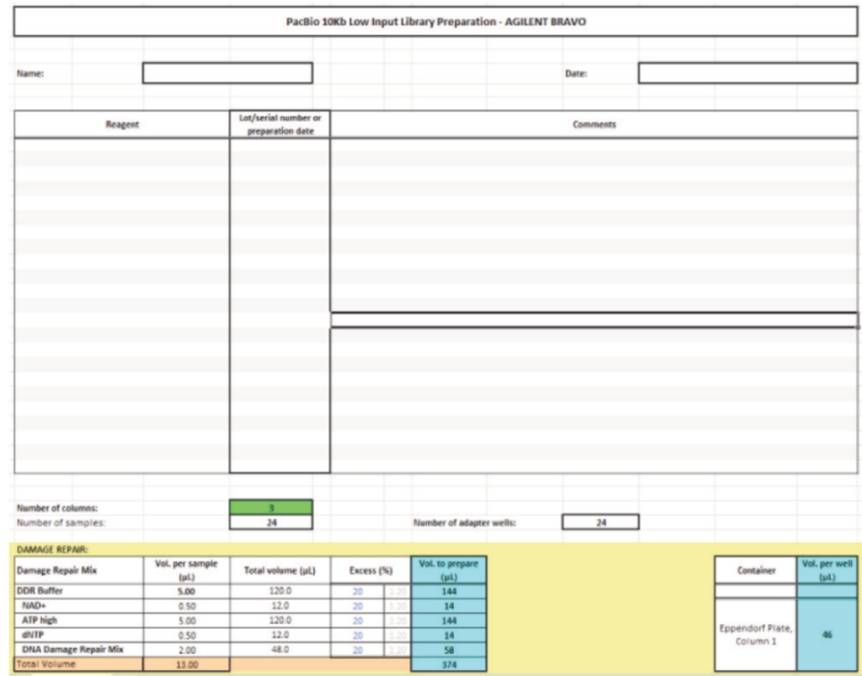

Fig. 2 Works protocols and Excel workbook for PacBio Library Preparation. WWorks protocols and Excel workbook for PacBio Library Preparation method provide an interactive, visual layout for the end user. a Post Shearing Cleanup Form. b 10 kb Library Prep Runset Dual SPRI Form. c PacBio Library Excel Workbook 


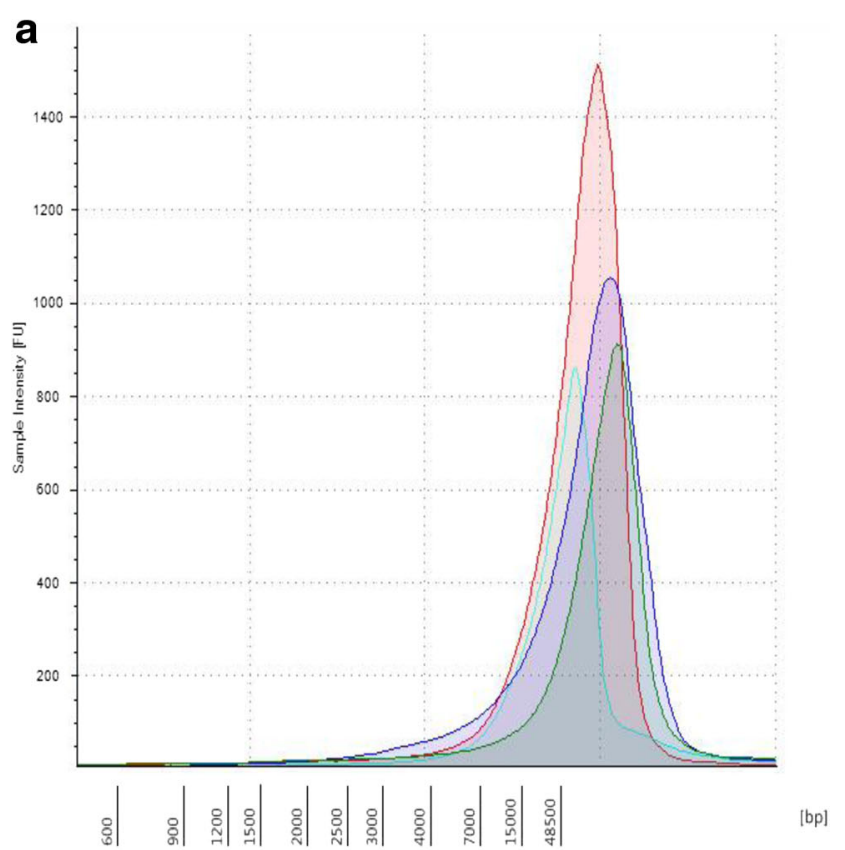

b $[$ bp]

$12 \quad 3 \quad 4$

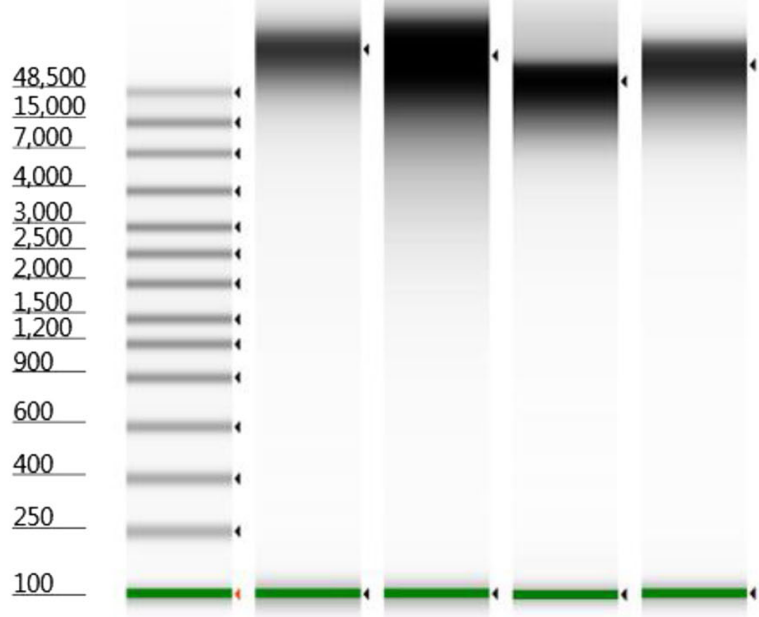

\section{DIN DIN DIN DIN \\ $\begin{array}{llll}9.1 & 9.1 & 9.3 & 9.5\end{array}$}

Fig. 3 Quantitation of Genomic DNA. Electropherogram (a) and gel image (b) of high molecular weight gDNA from Agilent 2200 TapeStation using the Genomic DNA ScreenTape System. Campylobacter (green), Listeria (blue), Vibrio (aqua), and Salmonella (red). Green lines at the bottom of the gel image are internal standards added to permit quantitation. Lower marker is not shown in the electropherogram 


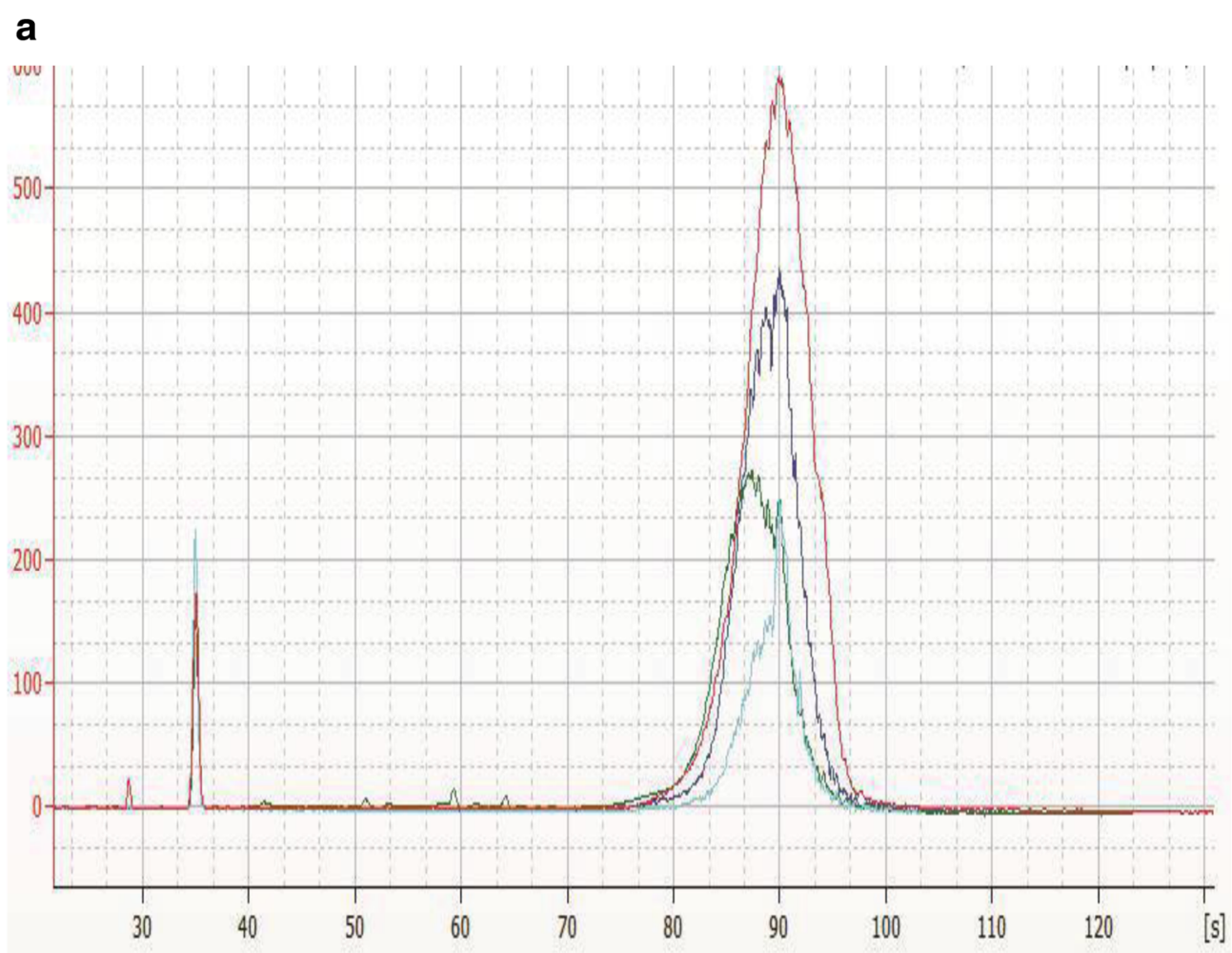

b

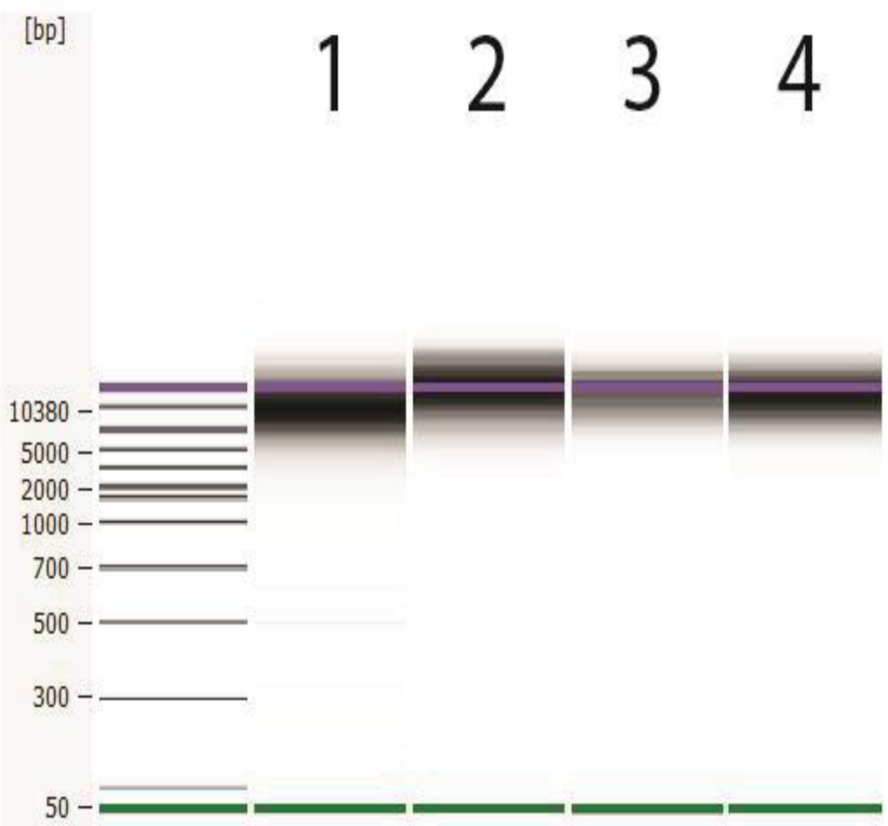

Fig. 4 Appearance of sheared DNA from Agilent 2100 Bioanalyzer analysis. Representative electropherogram (a) and virtual gel (b) are used for visual inspection (generated with the Agilent 2100 Bioanalyzer system with the DNA 12000 Kit) of sheared bacterial genomic DNA with average shearing size for Campylobacter (green, $10 \mathrm{~kb}$ ), Listeria (blue, $13.5 \mathrm{~kb}$ ), Vibrio (aqua,11.6 kb), and Salmonella (red, $17 \mathrm{~kb}$ ). Peaks near 35 are the lower marker internal standard for the DNA 12000 kit. A typical electropherogram using the Agilent Bioanalyzer 2100 DNA 12000 kit shows the lower marker at $35 \mathrm{~s}$ and the upper marker at $90 \mathrm{~s}$. The sheared DNA and the red upper marker, seen in the gel image, co-elute together 


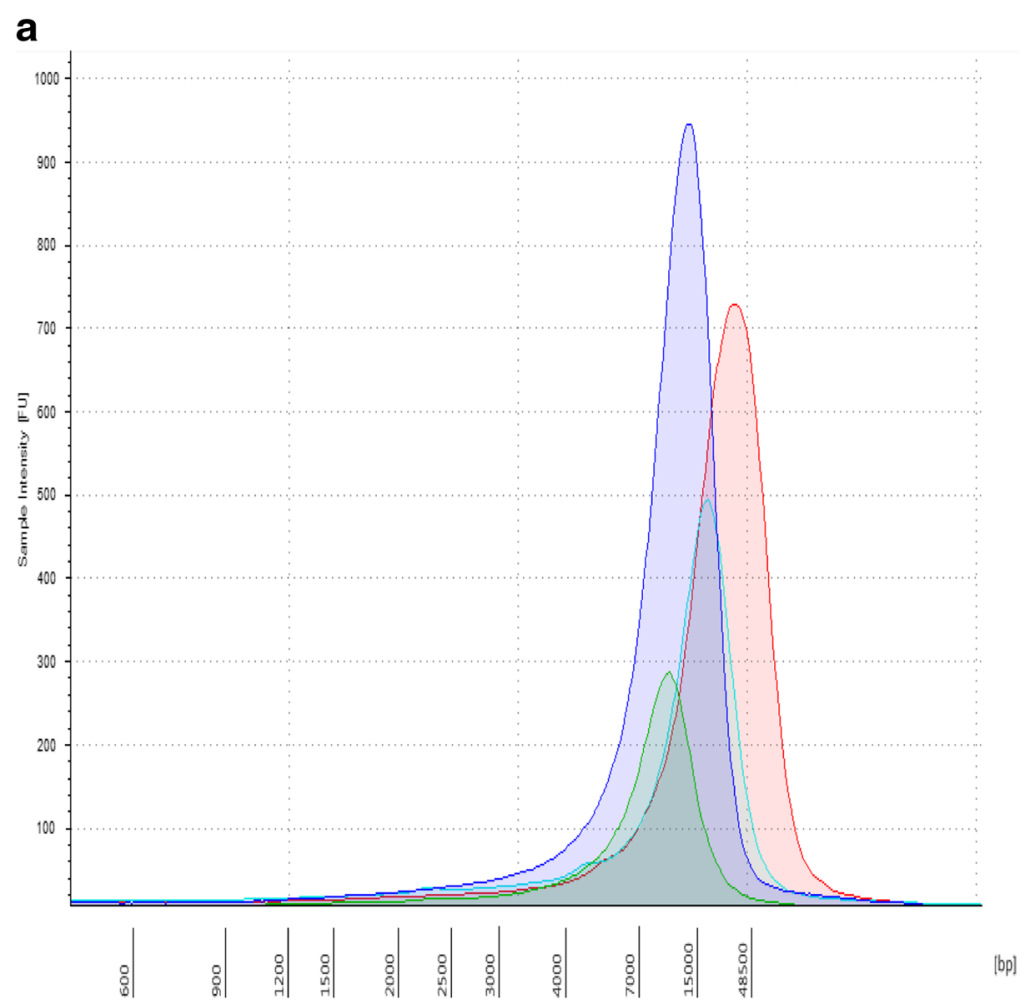

b

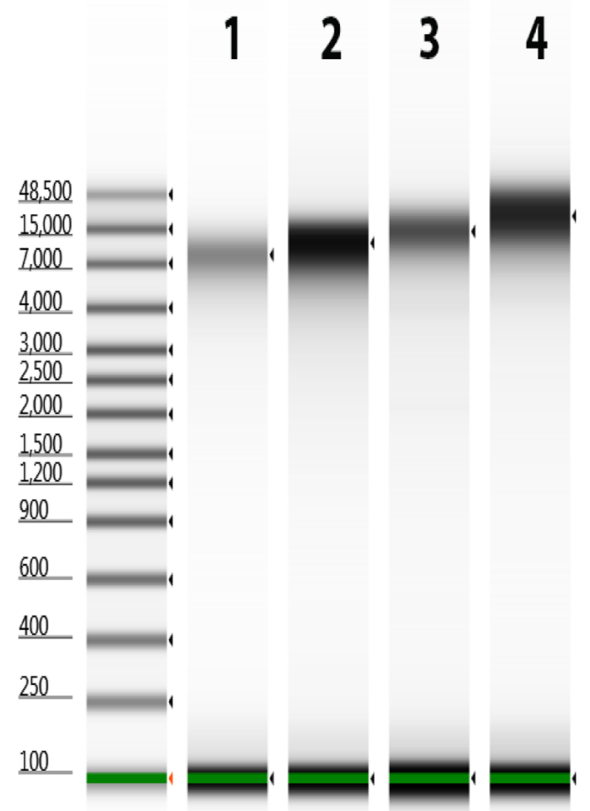

Fig. 5 Appearance of sheared DNA from Agilent 2200 TapeStation analysis. Representative electropherogram (a) and virtual gel (b) of sheared bacterial genomic DNA was generated with the Agilent 2200 TapeStation genomic DNA Kit with the average shearing size for Campylobacter (green, $16 \mathrm{~kb}$ ), Listeria (blue, $12 \mathrm{~kb}$ ), Vibrio (aqua, $14 \mathrm{~kb}$ ), and Salmonella (red, $20 \mathrm{~kb})$. Green lines at the bottom of the gel image are internal standards added to permit quantitation. Lower marker is not shown in the electropherogram 


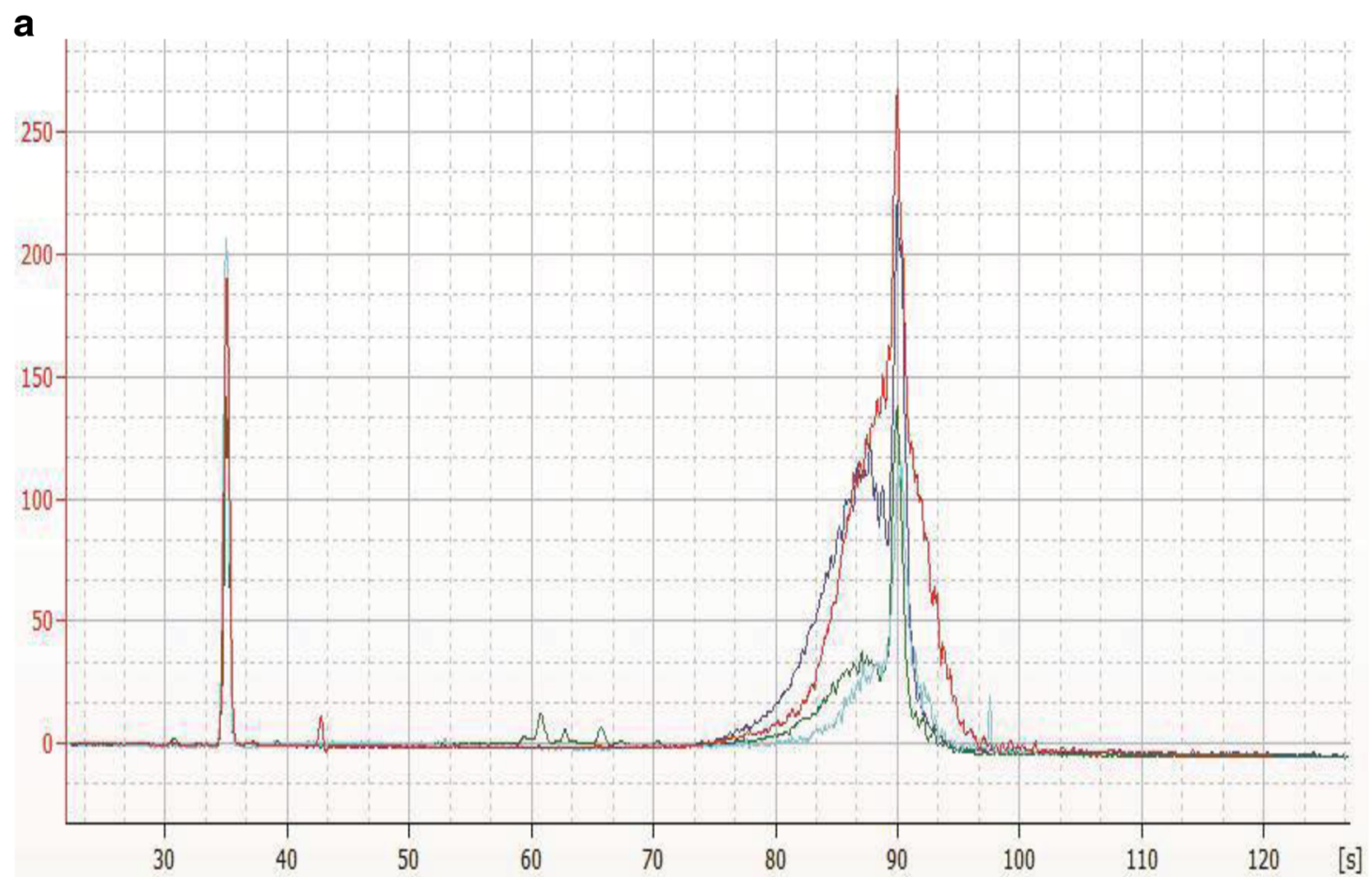

b

[bp]
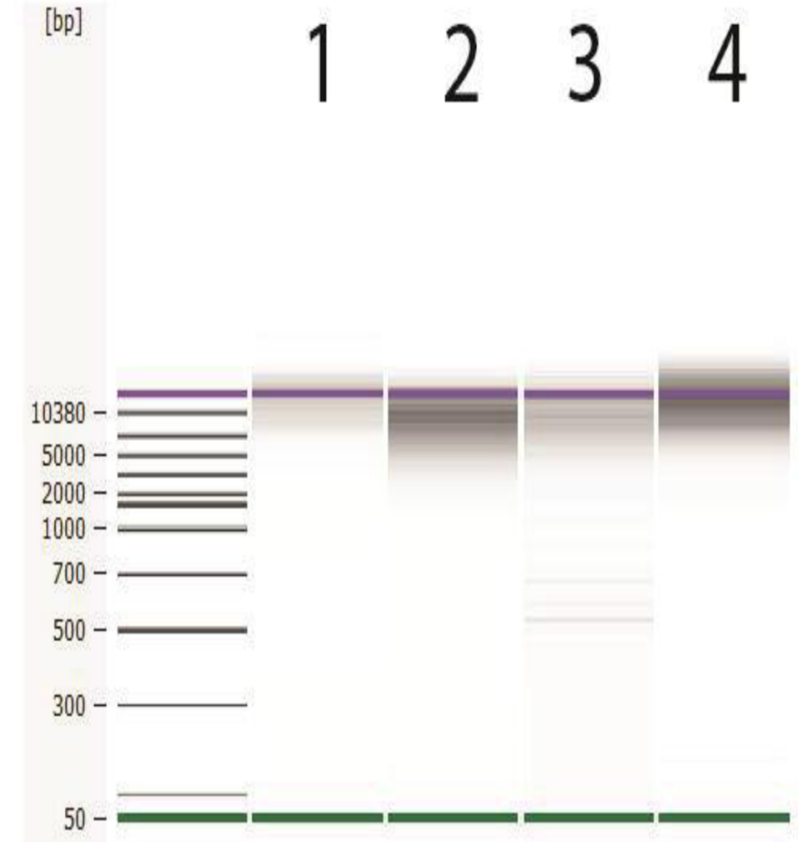

Fig. 6 Appearance of DNA libraries from Agilent 2100 Bioanalyzer analysis. Representative electropherogram (a) and virtual gel (b) used for visual inspection (generated with the Agilent 2100 Bioanalyzer system with the DNA $12000 \mathrm{Kit}$ ) of DNA libraries sizes prepared for sequencing with the PacBio SMRTbell $10 \mathrm{~kb}$ Template Preparation Kit on the Agilent NGS Workstation. A typical electropherogram using the Agilent bioanalyzer 2100 DNA 12000 kit shows the lower marker at $35 \mathrm{~s}$ and the upper marker at $90 \mathrm{~s}$. The DNA libraries and the upper marker co-elutes with each other, the sharper peak is the upper marker, shown in red on the gel image. The average library sizes are: Campylobacter (green, 9.1 kb), Listeria (blue, $9.5 \mathrm{~kb}$ ), Vibrio (aqua, $10 \mathrm{~kb}$ ), and Salmonella (red, $15 \mathrm{~kb}$ ) 


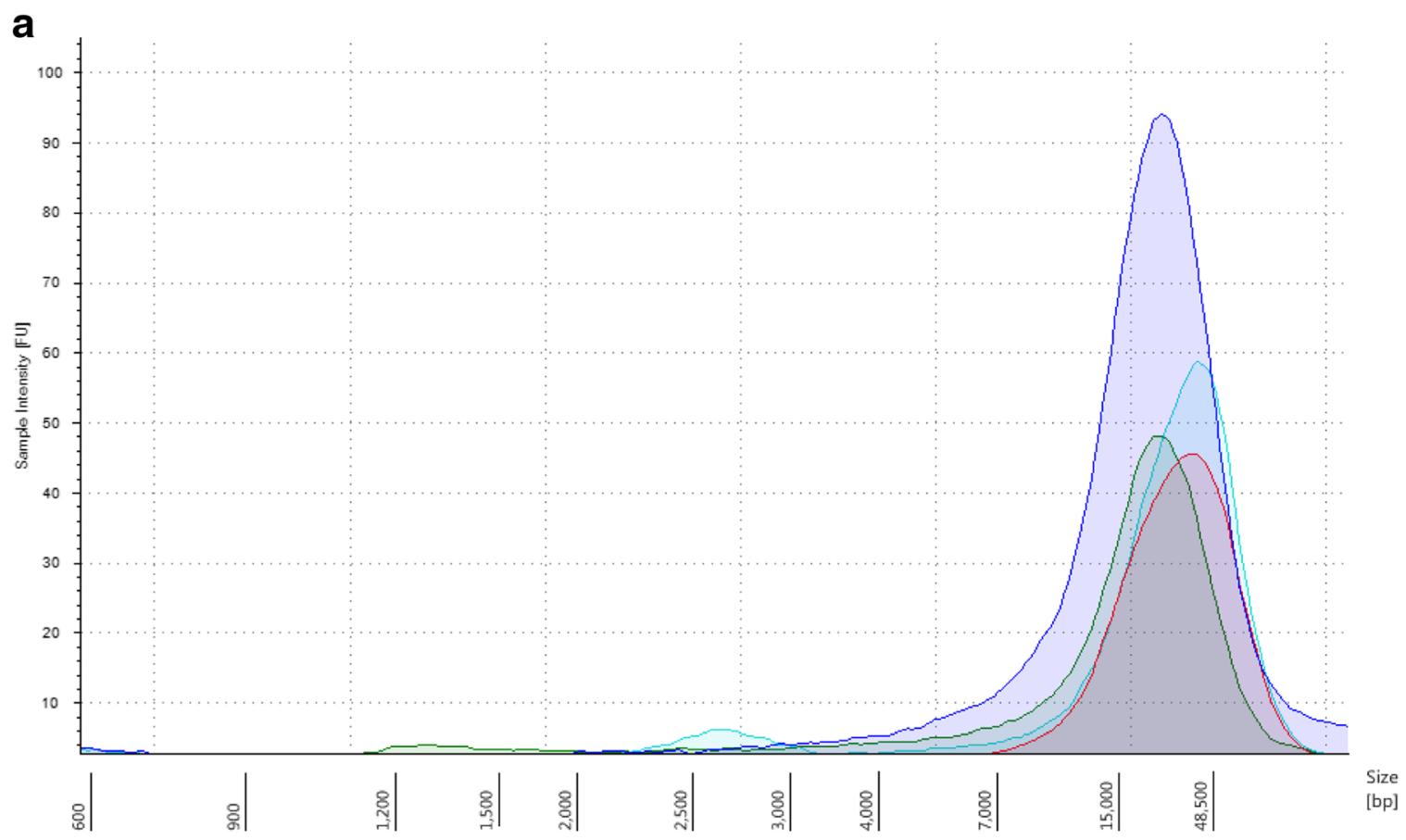

b

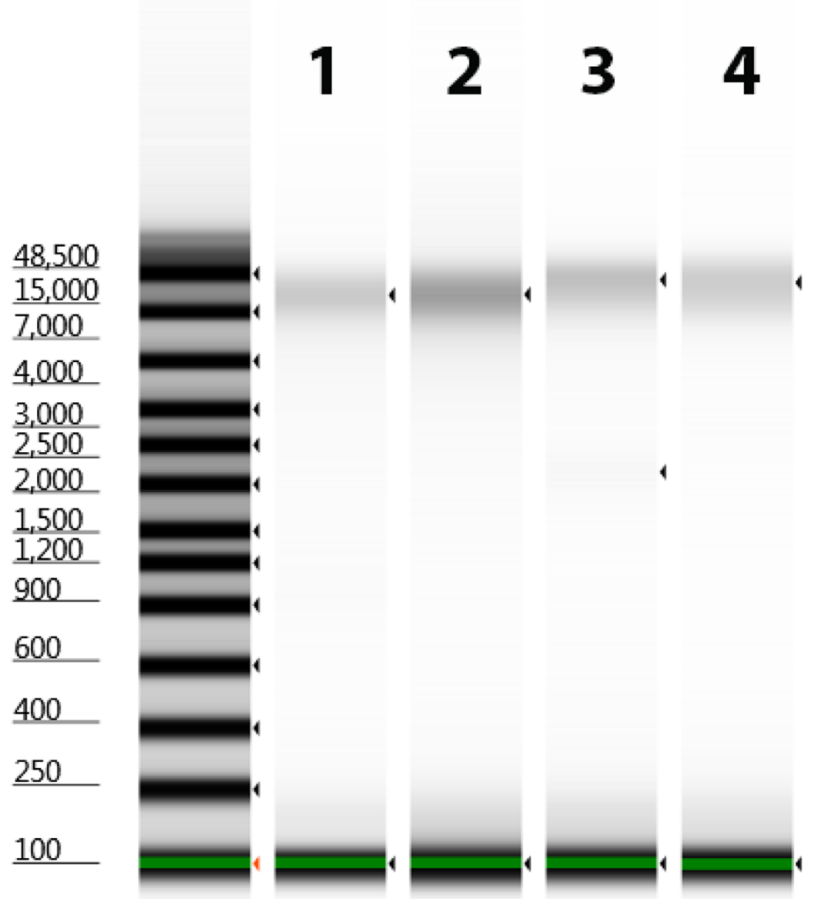

Fig. 7 Appearance of DNA libraries from Agilent 2200 TapeStation analysis. Representative electropherogram (a) and virtual gel (b) of DNA libraries sizes (generated with the Agilent 2200 TapeStation DNA genomics Kit) prepared for sequencing with the PacBio SMRTbell 10kb Template Preparation Kit on the Agilent NGS Workstation. The average library size for Campylobacter (green, 16 kb), Listeria (blue, 12 kb), Vibrio (aqua, 14 kb), and Salmonella (red, $20 \mathrm{~kb}$ ) is displayed on the software screen. Green lines at the bottom of the gel image are internal standards added to permit quantitation. Lower marker is not shown in the electropherogram 
shown in Fig. 3, where an electropherogram overlay and virtual gel images are shown for the four model organisms, together with the DIN calculated by the TapeStation software. The DNA Integrity Number (DIN) helped establish a cut-off for the suitability of the gDNA for further work and can be useful for library construction.

Following qualification of the gDNA, the next step is to shear the gDNA into the target fragment size required for library construction using a Covaris g-TUBE device according to manufacturer instructions. It is important to check the fragment size and the DNA amount prior to proceeding with the library construction. Traditionally, this has been done with the Agilent 2100 Bioanalyzer system with the DNA 12000 kit and these results are shown in Fig. 4 as overlaid electropherograms and a virtual gel image together with the sizing ladder provided. The DNA 12000 kit uses both a lower and an upper marker as internal standard. For these samples with a target size of $10 \mathrm{~kb}$, the DNA fragments usually run together with the upper marker, which can be easily seen on the gel image since it is shown in red. In the electropherogram view, the upper marker is the sharp peak at $90 \mathrm{~s}$. The Agilent 2200 TapeStation System with the gDNA ScreenTape assay can qualify the fragment size too, and this is shown in Fig. 5. The assay has a larger range to quantify genomic DNA larger than $12 \mathrm{~kb}$ with no upper marker and can run directly out of a 96 well plate. It is important to determine the correct sizing, in order for the sequencing facility to properly load the libraries on the sequencer.

Libraries are made following the PacBio SMRTbell 10kb Library Preparation on the Agilent NGS Workstation and traditionally confirmed with the Agilent 2100 Bioanalyzer System with the DNA 12000 kit, shown in Fig. 6. Thus, with SMRTbell templates around $10 \mathrm{~kb}$ in size, it's difficult to determine the correct sizing for those libraries as these constructs also run with the upper marker shown in red on the virtual gel images. Since the Agilent 2200 TapeStation System can size larger fragments up to $60 \mathrm{~kb}$, it can determine the size more accurately, as shown in Fig. 7.

\section{Conclusion}

The PacBio SMRTbell $10 \mathrm{~kb}$ Library preparation kit can be used with automation such as the Agilent Bravo to prepare microbial libraries with minimal GC bias. QC of the starting DNA and the required fragment preparation with the Covaris g-TUBE can be done with the Agilent 2200 TapeStation and the gDNA ScreenTape assay directly from the 96 well plates used by the Bravo to prepare the libraries.

\section{Abbreviations \\ DIN: DNA integrity number; gDNA: Genomic DNA; NGS: Next generation sequencing; SMRT: Single molecule, real-time}

\section{Acknowledgements}

We gratefully acknowledge the technical assistance provided by Kerry Le Sum Leung, Christina Kong, Lucy Cai, Alvin Leonardo, Vivian Lee, Surene
Foutouhi and Patrick Ancheta. We thank the 100K Pathogen Genome Sequencing Project for providing the cultures to conduct the study.

\section{Funding \\ Funding provided to BCW (NIH - 1R01HD065122-01A1; NIH - U24-DK097154; AGILENT TECHNOLOGIES THOUGHT LEADER AWARD, FDA - 5U01FD003572-04).}

Availability of data and materials

All data was analyzed during this study are included in this published article.

\section{Authors' contributions}

NK isolated DNA, conducted experiments, analyzed TapeStation data, and wrote the manuscript; $\mathrm{WN}$ and $\mathrm{KT}$ conducted experiments and analyzed TapeStation data; RA \& AW isolated DNA; KSK, JK and LH provided technical assistance with library preparation; LK conceived of experiments, analyzed data, and wrote the manuscript; SL provided programming of the automation to run the protocols; BCW conceived of experiments, analyzed data, and wrote the manuscript. All authors read and approved the final manuscript.

\section{Competing interests}

Agilent Technologies provided test instruments and initial funding to BCW. Pacific Biosciences provided PacBio SMRTBell 10 kb Library Preparation Kit and sequencing.

Consent for publication

Not applicable.

Ethics approval and consent to participate

Not applicable.

\section{Publisher's Note}

Springer Nature remains neutral with regard to jurisdictional claims in published maps and institutional affiliations.

\section{Author details}

${ }^{1}$ Population Health and Reproduction Department, School of Veterinary Medicine, University of California-Davis, Davis, CA, USA. ${ }^{2}$ Genentech, S. San Francisco, CA, USA. ${ }^{3}$ University of California-San Francisco, San Francisco, CA, USA. ${ }^{4}$ Pacific Biosciences, Menlo Park, CA, USA. ${ }^{5}$ Agilent Technologies, Inc., Santa Clara, CA, USA.

Received: 2 July 2016 Accepted: 26 February 2017

Published online: 23 March 2017

\section{References}

1. 100K Pathogen Genome Project. 2013 [cited 2016 June 30]; Available from: http://www.100kgenomes.org.

2. Kong N, et al. Production and analysis of high molecular weight genomic DNA for NGS pipelines using Agilent DNA extraction kit (p/n 200600). 2013. doi:10.13140/RG.2.1.2961.4807.

3. QIAamp DNA Mini Kit. 2016 [cited 2016 June 30]; Available from: https:// www.qiagen.com/us/shop/sample-technologies/dna/dna-preparation/ QIAamp-DNA-Mini-Kit\#resources.

4. Greenspoon SA, et al. QlAamp spin columns as a method of DNA isolation for forensic casework. J Forensic Sci. 1998;43(5):1024-30.

5. Agilent Genomic DNA ScreenTape System Quick Guide (p/n G2964-90040). 2016 [cited 2016 June 30]; Available from: http://www.agilent.com/cs/ library/usermanuals/Public/ScreenTape gDNA_OG.pdf.

6. Agilent 2200 TapeStation User Manual (p/n G2964-90002). 2016 [cited 2016 June 30]; Available from: http://www.agilent.com/cs/library/usermanuals/ Public/G2964-90002_TapeStationPalpatine_USR_EN.pdf.

7. Covaris. Covaris USER MANUAL: g-TUBE 2012 [cited 2016 June 30]; Available from: http://covarisinc.com/wp-content/uploads/pn_010154.pdf.

8. Agilent 2100 Bioanalyzer User Manual (p/n G2946-90004). 2016 [cited 2016 June 30]; Available from: https://www.agilent.com/cs/library/usermanuals/ Public/G2946-90004_Vespucci_UG_eBook_(NoSecPack).pdf.

9. Agilent Technologies, I. Agilent DNA 7500 and DNA 12000 Kit Quick Start Guide. 2013 [cited 2016 June 30]; Available from: http://www.agilent.com/ cs/library/usermanuals/Public/G2938-90025_DNA7500-12000_QSG.pdf. 